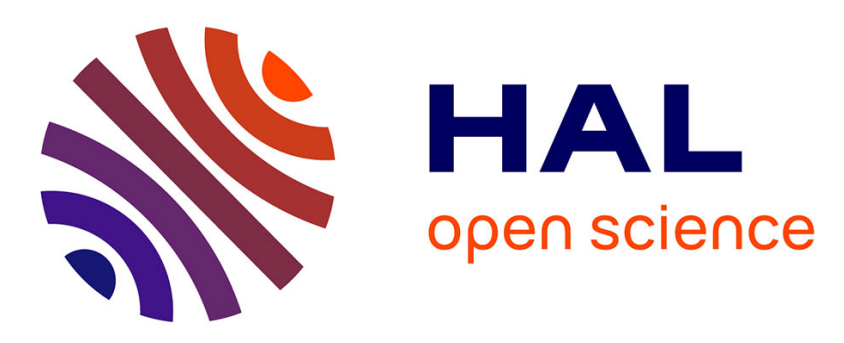

\title{
Exponential stabilization of language constrained discrete-time switched linear systems: A geometrical approach
}

\author{
Mirko Fiacchini, Marc Jungers, Antoine Girard
}

\section{To cite this version:}

Mirko Fiacchini, Marc Jungers, Antoine Girard. Exponential stabilization of language constrained discrete-time switched linear systems: A geometrical approach. ECC 2016 - 15th European Control Conference, Jun 2016, Aalborg, Denmark. pp.2035-2040, 10.1109/ecc.2016.7810591 . hal-01294210

\author{
HAL Id: hal-01294210 \\ https://hal.science/hal-01294210
}

Submitted on 22 Jul 2020

HAL is a multi-disciplinary open access archive for the deposit and dissemination of scientific research documents, whether they are published or not. The documents may come from teaching and research institutions in France or abroad, or from public or private research centers.
L'archive ouverte pluridisciplinaire HAL, est destinée au dépôt et à la diffusion de documents scientifiques de niveau recherche, publiés ou non, émanant des établissements d'enseignement et de recherche français ou étrangers, des laboratoires publics ou privés. 


\title{
Exponential stabilization of language constrained discrete-time switched linear systems: a geometrical approach
}

\author{
Mirko Fiacchini ${ }^{1}$, Marc Jungers ${ }^{2}$ and Antoine Girard ${ }^{3}$
}

\begin{abstract}
In this paper, the stabilizability of discrete-time linear switched systems subject to constraints on the switching law is considered. The admissible switching sequences are given by the language generated by a nondeterministic finite state automaton. An algorithm is proposed for computing contractive sets defined in the space of the continuous and finite states that induce recurrent exponential control Lyapunov functions for the system. A necessary and sufficient condition for the existence of recurrent control Lyapunov functions is given in terms of finite termination of the algorithm.
\end{abstract}

\section{INTRODUCTION}

Switched systems are systems whose behavior evolves among a finite class of different dynamics, [10]. Every behavior is characterized by the active mode that is selected by a function of time, the switching law. The interest in switched systems increased in the last decades due to their capability of modelling complex systems, such as embedded and networked systems. On the other hand, the analysis and control design for switched system might be rather involved, also for linear switched systems, [10], [15].

A classification of the switched systems might be done in terms of the assumptions on the switching law. It can be considered either as an arbitrary function, leading to robust stability analysis problems, or as a control function, leading to the problem of stabilizability and switching control design.

In this paper we are considering the problem of stabilizability of switched linear systems subject to constraints on the switching law. In many practical cases, indeed, the mode sequence might be required to satisfy some physical conditions. Consider for instance the problems of safety specifications, the tasks scheduling, the interaction between control and software implementation and the constraints on dwell-time switching. Several kinds of these constraints may be modeled by a nondeterministic finite automaton, see [2]. Roughly speaking, the switching law belongs to the language generated by such an automaton.

This work was partially supported by the ANR under the grant COMPACS (ANR-13-BS03-0004) and by the LabEx PERSYVAL-Lab (ANR-11LABX-0025-01) exploratory project 2014 "Set Theory and Algorithms for Dynamical Systems".

${ }^{1}$ M. Fiacchini is with Univ. Grenoble Alpes, GIPSA-lab, F-38000 Grenoble, France and CNRS, GIPSA-lab, F-38000 Grenoble, France. mirko.fiacchini@gipsa-lab.fr

${ }^{2} \mathrm{M}$. Jungers is with Université de Lorraine, CRAN, UMR 7039, 2 avenue de la forêt de Haye, Vandœuvre-lès-Nancy Cedex, 54516, France and CNRS, CRAN, UMR 7039, France and has been supported by Région Lorraine. marc.jungerseuniv-lorraine.fr

3 A. Girard is with the Laboratoire des signaux et systèmes (L2S), CNRS, CentraleSupélec, Université Paris-Sud, Université ParisSaclay, 3, rue Joliot-Curie, 91192 Gif-sur-Yvette, cedex, France Antoine.Girardel2s.centralesupelec.fr
Concerning the problem of stabilizability of switched system, it is known that convex Lyapunov functions lead to conservative results, and nonconvex ones must be considered, see [5]. Nonconvex Lyapunov functions induced by the union of ellipsoids are employed in [8], [18], [6], while more general homogeneous functions have been considered in [7].

The idea of employing regular languages and automata to impose constraints on the switching law has been recently applied to the problem of stability analysis for switched linear systems. The problem of stability of constrained switched linear system is addressed in [17], [16] using automata properties while converse Lyapunov theorems, based the joint spectral radius approach, are provided in [12]. Graph Lyapunov functions and spectral radius are employed in [1] and in [9] directed graphs are used to determine the switching sequences under which the system is stable.

Nevertheless, the problem of determining stabilizing feedback control policies satisfying language constraints, dealt with in this paper, has not been treated in the authors knowledge. For this purpose, we consider the nonconvex star-shaped sets, see [14], and their gauge functions as Lyapunov candidates, as in [7], to provide a geometric condition for stabilizability. An algorithm is provided whose finite termination is proved to be necessary and sufficient for the existence of a class of stabilizing control policy. This class of control policies, and the related nonconvex Lyapunov functions, are such that at least one state of the automaton is attained recurrently. A preliminary comparative analysis of the proposed stabilizability condition with respect to the general one and the periodic stabilizability is presented.

The paper is organized as follows. The Section II presents the problem; Section III provides the main results, concerning the algorithm and the condition for the existence of recurrent control Lyapunov functions. Examples are given in Section IV and Section V draws the conclusions.

Notations: Denote with $\mathbb{R}^{+}$the set on nonnegative real numbers. Given $n \in \mathbb{N}$, define $\mathbb{N}_{n}=\{j \in \mathbb{N}: 1 \leq j \leq n\}$. Given $\Omega \subseteq \mathbb{R}^{n}$ define the interior of $\Omega$ as $\operatorname{int}(\Omega)$ and its boundary as $\partial \Omega$. The Euclidean-norm in $\mathbb{R}^{n}$ is $\|x\|$ and the unit ball is denoted $\mathcal{B}^{n}$. The $i$-th element of a finite set of matrices is denoted as $A_{i}$. The set of $q$ switching modes is $\mathcal{I}=\mathbb{N}_{q}$, all the possible sequences of modes of length $N$ is $\mathcal{I}^{N}=\prod_{j=1}^{N} \mathcal{I}$ and $|\sigma|=N$ if $\sigma \in \mathcal{I}^{N}$.

\section{Problem Formulation}

Consider the discrete-time switched linear system

$$
x_{k+1}=A_{\sigma_{k}} x_{k},
$$


with $x_{k} \in \mathbb{R}^{n}$ the state at time $k \in \mathbb{N} ; \sigma: \mathbb{N} \rightarrow \mathcal{I}$ the switching law and $\left\{A_{i}\right\}_{i \in \mathcal{I}}$, with $A_{i} \in \mathbb{R}^{n \times n}$ for all $i \in \mathcal{I}$.

Assumption 1: The matrices $A_{i}$, with $i \in \mathcal{I}$, are nonsingular.

We impose the constraint that $\sigma$ has to belong to the language specified by a nondeterministic finite automaton.

Definition 1: A nondeterministic finite automaton is a tuple $\mathcal{A}=\left(\mathcal{S}, \mathcal{I}, \delta, \mathcal{S}_{0}\right)$ where $\mathcal{S}$ is a finite set of states, $\mathcal{I}=\mathbb{N}_{q}$ is a finite alphabet, $\delta: \mathcal{S} \times \mathcal{I} \rightarrow 2^{\mathcal{S}}$ is a set-valued transition map, and $\mathcal{S}_{0} \subseteq \mathcal{S}$ is a subset of initial states.

A state $s \in \mathcal{S}$ is reachable from $r \in \mathcal{S}$ if $s=r$ or if there exists a finite sequence $s_{0}, s_{1}, \ldots, s_{N} \in \mathcal{S}$ such that $s_{0}=r, s_{N}=s$ and for all $k \in \mathbb{N}_{N}$, there is $i_{k-1} \in \mathcal{I}$ such that $s_{k} \in \delta\left(s_{k-1}, i_{k-1}\right)$. A state $s \in \mathcal{S}$ is non-blocking if there is $i \in \mathcal{I}$ such that $\delta(s, i) \neq \emptyset$, it is blocking otherwise. A switching law $\sigma: \mathbb{N} \rightarrow \mathcal{I}$ belongs to the language of $\mathcal{A}$, denoted $L(\mathcal{A})$, if there is $s^{\sigma}: \mathbb{N} \rightarrow \mathcal{S}$ such that $s_{0}^{\sigma} \in \mathcal{S}_{0}$ and $s_{k+1}^{\sigma} \in \delta\left(s_{k}^{\sigma}, \sigma_{k}\right)$ for all $k \in \mathbb{N}$. Given $\Omega \subseteq \mathbb{R}^{n}$ and $s \in \mathcal{S}$, we denote the set $\Omega \times\{s\}$ as $\Omega \times s$ to simplify the notation.

A control policy $\nu: \mathbb{R}^{n} \times \mathcal{S} \rightarrow \mathcal{I} \times \mathcal{S}$, is such that

$$
\begin{gathered}
\nu(x, r)=(i(x, r), s(x, r)) \in \mathcal{I} \times \delta(r, i(x, r)), \\
\text { with } \delta(r, i(x, r)) \neq \emptyset .
\end{gathered}
$$

Moreover we denote with $\left(x_{N}^{\nu}\left(x_{0}, r_{0}\right), r_{N}^{\nu}\left(x_{0}, r_{0}\right)\right) \in \mathbb{R}^{n} \times$ $\mathcal{S}$ the state of the system (1) and automaton $\mathcal{A}$ at time $N$ starting from $\left(x_{0}, r_{0}\right)$ by applying the control policy $\nu$. Analogously, given $\sigma \in L(\mathcal{A})$ we denote with $x_{N}^{\sigma}\left(x_{0}\right)$ the state of (1) at time $N$ starting at $x_{0}$ under the switching sequence $\sigma$. The dependence of $x_{N}^{\nu}, r_{N}^{\nu}$ and $x_{N}^{\sigma}$ on the initial conditions will be dropped to simplify the notation. We denote, with slight abuse of notation, $\nu \in L(\mathcal{A})$ if every mode sequence $\sigma$ generated by $\nu$ is in $L(\mathcal{A})$. Finally $\sigma \in \mathcal{I}^{N}$ belongs to $L(\mathcal{A})$ if it is the prefix of an element of $L(\mathcal{A})$.

In the paper, we make the following assumptions on $\mathcal{A}$ :

Assumption 2: For all $s \in \mathcal{S}, s$ is non-blocking and there exists $s_{0} \in \mathcal{S}_{0}$ such that $s$ is reachable from $s_{0}$.

The assumption above is not restrictive since it is always possible to build from $\mathcal{A}$ an automaton $\mathcal{A}^{\prime}$ satisfying Assumption 2 and such that $L(\mathcal{A})=L\left(\mathcal{A}^{\prime}\right)$. Indeed, $\mathcal{A}^{\prime}$ is obtained firstly by removing all the states not reachable from an initial state and secondly by removing iteratively all blocking states. Remark also that, rigorously speaking, Definition 1 is not the classical definition of nondeterministic finite automaton since we do not define a set of final states, as we consider infinite sequences. Definition 1 actually defines a subclass of nondeterministic Büchi automaton, see [2].

Remark 1: The stabilizability of the system (1) subject to the language generated by $\mathcal{A}$ can be reduced to the that of (1) subject to $L\left(\mathcal{A}^{\prime}\right)$, with $\mathcal{A}^{\prime}=(\mathcal{S}, \mathcal{I}, \delta, \mathcal{S})$. In fact, from Assumption 2, they have the same trajectories and languages, except an initial finite transient required to $s_{0} \in \mathcal{S}_{0}$ to reach any $s \in \mathcal{S}$. Then, in the paper we will consider that $\mathcal{S}_{0}=\mathcal{S}$.

We consider here the following notion of stabilizability:

Definition 2: The system (1) is globally exponentially stabilizable relatively to the language $L(\mathcal{A})$ if there are $c \geq 0$ and $\lambda \in[0,1)$ and, for all $x \in \mathbb{R}^{n}$, there exists a switching law $\sigma \in L(\mathcal{A})$, such that

$$
\left\|x_{k}^{\sigma}(x)\right\| \leq c \lambda^{k}\|x\| .
$$

We also give below the definition of exponentially stabilizing control Lyapunov function for switched systems, adapted from that one formulated in [18].

Definition 3: A nonnegative continuous function $V: \mathbb{R}^{n} \times$ $\mathcal{R} \rightarrow \mathbb{R}^{+}$is an exponentially stabilizing control Lyapunov function (ECLF) of system (1) in $\mathcal{R} \subseteq \mathcal{S}$, with $\mathcal{R}$ non-empty, if for every $(x, r) \in \mathbb{R}^{n} \times \mathcal{R}$, we have

(i) $\kappa_{1}\|x\| \leq V(x, r) \leq \kappa_{2}\|x\|$ for some finite positive constants $\kappa_{1}$ and $\kappa_{2}$

(ii) $V(x, r)-V\left(x_{1}^{\nu}, r_{1}^{\nu}\right) \geq \kappa_{3}\|x\|$ for some constant $\kappa_{3}>$ 0 and control policy $\nu(x, r)$ as in (2) and such that $\delta(r, \nu(x, r)) \in \mathcal{R}$.

The existence of an ECLF implies exponential stabilizability of the system (1)

Remark 2: Note that it is sufficient that the ECLF as well as the control policy $\nu$ were defined on a subset of the automaton states $\mathcal{R}$, since every state is assumed in the set of initial states, see Assumption 1 and Remark 1, provided the automaton state is maintained in $\mathcal{R}$ under $\nu$.

We consider the particular case when the stabilizing switching law is such that the automaton reaches one state $s \in \mathcal{S}$ every at most $N$ steps. We will refer to this class of switching sequences as recurrent.

Definition 4: The automaton trajectory $r^{\sigma}: \mathbb{N} \rightarrow \mathcal{S}$ is ultimately recurrent in $s \in \mathcal{S}$ under the switching sequence $\sigma \in L(\mathcal{A})$ if there exist $m \in \mathbb{N}, N \in \mathbb{N}$ and a sequence $l_{k}$ : $\mathbb{N} \rightarrow \mathbb{N}$ such that $l_{1}=m$ and $r_{l_{k}}^{\sigma}=s$, and $1 \leq l_{k+1}-l_{k} \leq N$ for all $k \in \mathbb{N}$. It is recurrent in $s$ under $\sigma$ if it is ultimately recurrent with $m=0$.

We are interested in the family of functions that are ECLF under admissible automaton trajectories that are recurrent.

Definition 5: A nonnegative continuous function $V: \mathbb{R}^{n} \times$ $\mathcal{R} \rightarrow \mathbb{R}^{+}$is a recurrent exponentially stabilizing control Lyapunov function of system (1) in $\mathcal{R} \subseteq \mathcal{S}$ if it is an exponentially stabilizing control Lyapunov in $\mathcal{R}$ under a control policy $\nu$ as in (2) such that $\nu \in L(\mathcal{A})$ and it generates trajectories recurrent in a state $s \in \mathcal{R}$.

The C-sets, i.e. compact, convex sets containing the origin in their interior, and the induced gauge functions are widely employed for robust stability and stabilizability of parametric uncertain linear systems, [3], [4]. In this paper we employ analogous geometrical concepts. For this we recall that a compact set $\Omega$ is star-convex respect to $z \in \Omega$ if every convex combination of $x$ and $z$ belongs to $\Omega$ for all $x \in \Omega$, see [14].

Definition 6: A set $\Omega \subseteq \mathbb{R}^{n}$ is a $\mathrm{C}^{*}$-set if it is compact, star-convex with respect to the origin and $0 \in \operatorname{int}(\Omega)$. The gauge function of a $C^{*}$-set $\Omega \subseteq \mathbb{R}^{n}$ is

$$
\Psi_{\Omega}(x)=\min _{\alpha \geq 0}\{\alpha \in \mathbb{R}: x \in \alpha \Omega\} .
$$

Some basic properties of the $\mathrm{C}^{*}$-sets and their gauge functions are listed below, see also [14].

Property 1: Every C-set is a $\mathrm{C}^{*}$-set. Given a $\mathrm{C}^{*}$-set $\Omega \subseteq$ $\mathbb{R}^{n}$, we have that $\alpha \Omega \subseteq \Omega$ for all $\alpha \in[0,1]$, and the gauge function $\Psi_{\Omega}(x)$ is continuous; homogeneous of degree one, 
i.e. $\Psi_{\Omega}(\alpha x)=\alpha \Psi_{\Omega}(x)$ for all $\alpha \geq 0$ and $x \in \mathbb{R}^{n}$; positive definite; defined on $\mathbb{R}^{n}$ and radially unbounded. For every family of $\mathrm{C}^{*}$-sets $\Omega_{i}$ with $i \in \mathbb{N}_{I}$, also the sets $\underline{\Omega}=\bigcup_{i \in I} \Omega_{i}$ and $\bar{\Omega}=\bigcap_{i \in I} \Omega_{i}$ are $C^{*}$-sets and $\Psi_{\Omega}(x)=\min _{i \in \mathbb{N}_{I}} \Psi_{\Omega_{i}}(x)$ and $\Psi_{\bar{\Omega}}(x)=\max _{i \in \mathbb{N}_{I}} \Psi_{\Omega_{i}}(x)$. Moreover $\Psi_{\mathcal{B}^{n}}(x)=\|x\|$ and $\Omega_{i} \subseteq \Omega_{j}$ if and only if $\Psi_{\Omega_{j}}(x) \leq \Psi_{\Omega_{i}}(x)$ for all $x \in \mathbb{R}^{n}$. Finally, for every nonsingular matrix $A \in \mathbb{R}^{n \times n}$ and $\mathrm{C}^{*}$-set $\Omega \subseteq \mathbb{R}^{n}$, also $A \Omega$ is a $\mathrm{C}^{*}$-set in $\mathbb{R}^{n}$.

The gauge functions induced by $\mathrm{C}$-sets have been used in literature as Lyapunov functions candidates, for linear parametric uncertain systems, [13], [3], and switched systems with arbitrary switching, [11]. On the other hand, the gauge functions of $\mathrm{C}^{*}$-sets are proved to be a universal class of Lyapunov functions for switched systems with switching control law, see [7], [6]. We will be searching, then, for contractive $\mathrm{C}^{*}$-sets such that the related gauge functions could result to be recurrent ECLF for the switched systems subject to the language constraints induced by $\mathcal{A}$.

\section{NeCESSARY AND SufFicient Condition}

For every set $\Omega \subseteq \mathbb{R}^{n}$, state $s \in \mathcal{S}$ and mode $i \in \mathcal{I}$, define the one-step operator for the switched system (1) whose switching law is specified by $\mathcal{A}$ as

$$
Q_{i}^{s}(\Omega)=\left\{(x, r) \in \mathbb{R}^{n} \times \mathcal{S}: A_{i} x \in \Omega, \quad s \in \delta(r, i)\right\} .
$$

Namely, given a set $\Omega$, a state of the automaton $s$ and a mode $i$, the operator $Q_{i}^{s}(\Omega)$ gives

$$
Q_{i}^{s}(\Omega)=\bigcup_{r \in \gamma(s, i)}\left(A_{i}^{-1} \Omega \times r\right)
$$

where $\gamma(s, i)$ are the automaton states that evolve to $s$ by applying the mode $i$, i.e. $\gamma(s, i)=\{r \in \mathcal{S}: s \in \delta(r, i)\}$. Thus, intuitively, $Q_{i}^{s}(\Omega)$ is the pre-image through the mode $i \in \mathcal{I}$, in the space $\mathbb{R}^{n} \times \mathcal{S}$, of the set $\Omega \times s$. Clearly, if $s \notin \delta(r, i)$ for all $r \in \mathcal{S}$, then $Q_{i}^{s}(\Omega)=\emptyset$. Moreover define:

$$
Q^{s}(\Omega)=\bigcup_{i \in \mathcal{I}} Q_{i}^{s}(\Omega)=\bigcup_{i \in \mathcal{I}} \bigcup_{r \in \gamma(s, i)}\left(A_{i}^{-1} \Omega \times r\right),
$$

that is the set of the pre-images of the set $\Omega \times s$ through all the modes $i \in \mathcal{I}$. Consider the Algorithm 1.

- Initialization: given the $\mathrm{C}^{*}$-set $\Omega_{0} \subseteq \mathbb{R}^{n}$ and a state $s \in \mathcal{S}$, define $\Lambda_{0}^{s}=\Omega_{0} \times s$ and $k=0$;

- Iteration for $k \geq 0$ :

$$
\begin{aligned}
& \Lambda_{k+1}^{s}=\bigcup_{(\Omega \times r) \subseteq \Lambda_{k}^{s}} Q^{r}(\Omega), \\
& \Omega_{k+1}^{s, s}=\left\{x \in \mathbb{R}^{n}:(x, s) \in \Lambda_{k+1}^{s}\right\},
\end{aligned}
$$

- Stop if $\Omega_{0} \subseteq \operatorname{int}\left(\bigcup_{j \in \mathbb{N}_{k+1}} \Omega_{j}^{s, s}\right)$; denote $N^{s}=k+1$ and

$$
\Omega^{s}=\bigcup_{j \in \mathbb{N}_{N}} \Omega_{j}^{s, s} .
$$

Algorithm 1: Computation of a contractive $\mathrm{C}^{*}$-set for the system (1), satisfying Assumption 1, recurrent in $s$.
The geometrical interpretations of the sets $\Lambda_{k+1}^{s}$ and $\Omega_{k+1}^{s, s}$ follow, their dependence on $\Omega_{0}$ is avoided. First notice that the sets $\Lambda_{j}^{s}$ lie in the space $\mathbb{R}^{n} \times \mathcal{S}$, then they have the form

$$
\Lambda_{j}^{s}=\bigcup_{r \in \mathcal{S}}\left(\Omega_{j}^{r, s} \times r\right), \quad \forall j \in \mathbb{N},
$$

with $\Omega_{j}^{r, s}$ possibly empty. In particular one has

$$
\Omega_{j}^{r, s}=\left\{x \in \mathbb{R}^{n}:(x, r) \in \Lambda_{j}^{s}\right\}, \quad \Omega^{r, s}=\bigcup_{j \in \mathbb{N}_{N^{s}}} \Omega_{j}^{r, s} .
$$

The index $s$ denotes the initial automaton state. Given $\Lambda_{k}^{s} \in$ $\mathbb{R}^{n} \times \mathcal{S}$, the points in $\mathbb{R}^{n} \times \mathcal{S}$ that can be steered in one step in $\Lambda_{k}^{s}$, by means of an admissible mode, are the set $\Lambda_{k+1}^{s}$. Thus, $\Lambda_{k}^{s}$ is the set of $(x, r) \in \mathbb{R}^{n} \times \mathcal{S}$ that can be steered in $\Omega_{0} \times s$ by means of admissible sequences of modes of length $k$. The set $\Omega_{k}^{s, s}$ is, then, the set of states $x \in \mathbb{R}^{n}$ such that, if the automaton state is $s$, a trajectory exists that steers $x$ in $\Omega_{0}$ in $k$ steps, with the automaton state returning at $s$.

Lemma 1: The sets $\Omega_{k}^{r, s}$ are $\mathrm{C}^{*}$-sets or empty, for all $k \in$ $\mathbb{N}_{\mathbb{N}^{s}}$ and $r \in \mathcal{S}$.

Proof: The result comes directly from the properties of $\mathrm{C}^{*}$-sets, see Property 1.

The main result, providing a constructive method for determining whether the system (1) can be stabilized by means of a switching sequence in $L(\mathcal{A})$, is now presented.

Theorem 1: There exists a non-empty set $\mathcal{R} \subseteq \mathcal{S}$ and a recurrent exponential control Lyapunov function in $\mathcal{R}$ if and only if Algorithm 1 terminates in finite time for a state $s \in \mathcal{S}$.

Proof: First we prove sufficiency, that is the fact that the finite termination of the algorithm implies the existence of a recurrent ECLF in $\mathcal{R}$, with $\mathcal{R}$ non-empty. Suppose that the algorithm terminates with finite $N^{s} \in \mathbb{N}$ and define $\mathcal{R}=$ $\left\{r \in \mathcal{S}: \Omega^{r, s} \neq \emptyset\right\}$, non-empty since at least $s \in \mathcal{R}$, from $\Omega_{0} \subseteq \operatorname{int}\left(\Omega^{s}\right)$. From the geometrical meaning, $\Omega^{s}$ is the set of $x \in \mathbb{R}^{n}$ that can be driven to $\Omega_{0}$, with the automaton initial and final states equal to $s$, by means of an admissible switching sequence of length smaller or equal than $N^{s}$ that maintains the automaton state in $\mathcal{R}$. Moreover, since $\Omega_{0}$ and $\Omega^{s}$ are $\mathrm{C}^{*}$-sets, then there exists $\rho \in(0,1)$ such that

$$
\Omega_{0} \subseteq \rho \Omega^{s} \subseteq \Omega^{s},
$$

from $\Omega_{0} \subseteq \operatorname{int}\left(\Omega^{s}\right)$. Define $\mu \in\left(\rho^{1 /\left(N^{s}-1\right)}, 1\right)$ and the sets

$$
\Theta_{j}^{r, s}=\mu^{j} \Omega_{j}^{r, s}, \quad \Theta^{r, s}=\bigcup_{j \in \mathbb{N}_{N} s} \Theta_{j}^{r, s}, \quad \forall r \in \mathcal{R},
$$

with $\Omega_{j}^{r, s}$ as in (4), (5) and (6). Consider the function

$$
V(x, r)=\Psi_{\Theta^{r, s}}(x)=\min _{j \in \mathbb{N}_{N} s} \Psi_{\Theta_{j}^{r, s}}(x)=\min _{j \in \mathbb{N}_{N^{s}}} \mu^{-j} \Psi_{\Omega_{j}^{r, s}}(x),
$$

defined for all $(x, r) \in \mathbb{R}^{n} \times \mathcal{R}$. Condition (i) in Definition 3 holds since $\Theta^{r, s}$ are $\mathrm{C}^{*}$-sets and then there exist $\kappa_{1}^{r, s}, \kappa_{2}^{r, s}>$ 0 such that $\kappa_{2}^{r, s} \mathcal{B}^{n} \leq \Theta^{r, s} \leq \kappa_{1}^{r, s} \mathcal{B}^{n}$, which implies

$\kappa_{1}^{r, s}\|x\|=\kappa_{1}^{r, s} \Psi_{\mathcal{B}^{n}}(x) \leq \Psi_{\Theta^{r, s}}(x) \leq \kappa_{2}^{r, s} \Psi_{\mathcal{B}^{n}}(x)=\kappa_{2}^{r, s}\|x\|$.

To prove that also condition (ii) holds, consider $(x, r) \in$ $\mathbb{R}^{n} \times \mathcal{R}$ and denote, for notational convenience, $\Psi_{\Theta^{r, s}}(x)=$ 
$\alpha$ and $\left(x_{1}^{\nu}, r_{1}^{\nu}\right)=\left(x^{+}, r^{+}\right)$. Define

$$
\begin{aligned}
& j^{r, s}(x)=\min _{\sigma \in \mathcal{I}^{\left[1: N^{s}\right]}}\left\{j=|\sigma|: \exists m \in \mathcal{S}^{j+1} \quad\right. \text { s.t. } \\
& \quad m_{1}=r, \quad m_{j+1}=s, \quad m_{k+1} \in \delta\left(m_{k}, \sigma_{k}\right) \quad \forall k \in \mathbb{N}_{j}, \\
& \left.\Psi_{\Omega_{0}}\left(x_{j}^{\sigma}\right) \leq \Psi_{\Omega^{r, s}}(x), \quad \sigma \in L(\mathcal{A})\right\}
\end{aligned}
$$

that is in $\mathbb{N}_{N^{s}}$ since by construction of $\Omega^{r, s}$ there exists a sequence of modes in $L(\mathcal{A})$ of length smaller than or equal to $N^{s}$ that steers $r$ in $s$ and $x \in \Omega_{0}$, if $x \in \partial \Omega^{r, s}$. Note that from the definition above we have that

$$
x \in \partial\left(\alpha \Theta_{j^{r, s}}^{r, s}\right)=\partial\left(\alpha \mu^{j^{r, s}} \Omega_{j^{r, s}}^{r, s}\right),
$$

where the dependence of $j^{r, s}$ on $x$ has been avoided. Define the control policy $\nu(x, r)$ as the elements $\left(\sigma_{1}, m_{2}\right)$ with $\sigma$ and $m$ a selection among the optimizers of the minimization in (10) at $(x, r)$. Therefore, $j^{r^{+}, s}\left(x^{+}\right) \leq j^{r, s}(x)-1$ if $r^{+} \neq s$, which implies that $s$ is attained every $N^{s}$ steps at most.

Concerning $j^{r, s}$ in (10), either $j^{r, s}=1$ or $2 \leq j^{r, s} \leq N^{s}$. Consider first the case $j^{r, s}(x) \geq 2$. By construction, for all $\beta \geq 0$ and $2 \leq j \leq N^{s}$ and every $(x, r) \in \mathbb{R}^{n} \times \mathcal{R}$ we have $x \in \partial\left(\beta \Omega_{j}^{r, s}\right) \Rightarrow \exists(i, m) \in \mathcal{I} \times \delta(r, i)$ s.t $A_{i} x \in \partial\left(\beta \Omega_{j-1}^{m, s}\right)$.

Note that a possible choice for $(i, m)$ is the value of the control policy $\nu$ at $(x, r)$, i.e. $\nu(x, r)=(i, m)$. As noticed above, see (11) and (12), we have that $x \in \partial\left(\alpha \mu^{j^{r, s}} \Omega_{j^{r, s}}^{r, s}\right)$, and by applying the control $\nu(x, r)$, we have

$$
x^{+} \in \partial\left(\alpha \mu^{j^{r, s}} \Omega_{j^{r, s}-1}^{r^{+}, s}\right)=\partial\left(\alpha \mu \Theta_{j^{r, s}-1}^{r^{+}, s}\right) .
$$

Then we have

$$
\begin{aligned}
& V\left(x^{+}, r^{+}\right)=\Psi_{\Theta^{r+, s}}\left(x^{+}\right)=\min _{j \in \mathbb{N}_{N^{s}}} \Psi_{\Theta_{j}^{r+, s}}\left(x^{+}\right) \\
& \leq \Psi_{\Theta_{j^{r, s}-1}^{r+,}}\left(x^{+}\right) \leq \alpha \mu=\Psi_{\Theta^{r, s}}(x) \mu=\mu V(x, r),
\end{aligned}
$$

with $\mu \in(0,1)$, then $V(x, r)$ decreases by applying $\nu(x, r)$.

Consider now $j^{r, s}(x)=1$, which means that $x \in$ $\partial\left(\alpha \Theta_{1}^{r, s}\right)$, see (11). In this case, and by construction of $\Omega_{1}^{r, s}$, the control is $\nu(x, r)=(j, s)$ and then the successor can be in $\left(\alpha \mu \Omega_{0}\right) \times s$. Thus applying $\nu(x, r)$ we have $r^{+}=s$ and

$$
\begin{aligned}
& x^{+} \in \partial\left(\alpha \mu \Omega_{0}\right) \subseteq \alpha \mu \rho \Omega^{s}=\alpha \mu^{-N^{s}+1} \rho\left(\mu^{N^{s}} \Omega^{s}\right) \\
& \quad=\alpha \mu^{-N^{s}+1} \rho\left(\mu^{N^{s}} \bigcup_{j \in \mathbb{N}_{N^{s}}} \Omega_{j}^{s, s}\right) \subseteq \alpha \mu^{-N^{s}+1} \rho \bigcup_{j \in \mathbb{N}_{N^{s}}} \mu^{j} \Omega_{j}^{s, s} \\
& \quad=\alpha \rho \mu^{-N^{s}+1} \Theta^{s, s}
\end{aligned}
$$

from (7), which implies

$$
\begin{aligned}
& V\left(x^{+}, r^{+}\right)=\Psi_{\Theta^{s, s}}\left(x^{+}\right) \leq \rho \mu^{-N^{s}+1} \alpha \\
& \quad=\rho \mu^{-N^{s}+1} \Psi_{\Theta^{r, s}}(x)=\rho \mu^{-N^{s}+1} V(x, s) .
\end{aligned}
$$

Therefore $V(x, r)$ decreases, since $\rho \mu^{-N^{s}+1}<1$. Thus, denoting $\lambda=\max \left\{\mu, \rho \mu^{-N^{s}+1}\right\}$, we have that for every $(x, r) \in \mathbb{R}^{n} \times \mathcal{R}$ there exists a $\nu(x, r)$ as in (2) such that $V\left(x^{+}, r^{+}\right) \leq \lambda V(x, r)$, with $\lambda \in(0,1)$. Finally defining

$$
d=\min _{b>0}\left\{b \in \mathbb{R}: \Theta^{r, s} \subseteq b \mathcal{B}^{n}, \forall r \in S\right\}
$$

which exists finite since $\Theta^{r, s}$ are non-empty $\mathrm{C}^{*}$-sets, then $V(x, r)=\Psi_{\Theta^{r, s}}(x) \geq d\|x\|$, for all $x$ and $r$, it follows

$$
V(x, r)-V\left(x^{+}, r^{+}\right) \geq(1-\lambda) V(x, r) \geq(1-\lambda) d\|x\|,
$$

and then (ii) in Definition 3 holds with $\kappa_{3}=(1-\lambda) d$.

Concerning necessity, suppose that a recurrent ECLF in $\mathcal{R} \subseteq \mathcal{S}$ exists. This implies that the system is exponentially stabilizable under a control policy $\nu: \mathbb{R}^{n} \times \mathcal{R} \rightarrow \mathcal{I} \times \mathcal{R}$ such that $\nu \in L(\mathcal{A})$ and the trajectories are recurrent in $s$ and maintained in $\mathcal{R}$. Then, from Definition 2, we have that there exists $\nu(x, r)$ recurrent in $s$, with cycle length bounded by $N \in \mathbb{N}$, such that $\left\|x_{k}^{\nu}\right\| \leq c \lambda^{k}\|x\|$ for all $(x, r) \in \mathbb{R}^{n} \times \mathcal{R}$. Given the initial $\mathrm{C}^{*}$-set $\Omega_{0}$, fix $\tau \in(0,1)$ and define

$$
\begin{aligned}
& \alpha_{m}^{s}=\max _{a \geq 0}\left\{\alpha \in \mathbb{R}: \alpha \mathcal{B}^{n} \subseteq \Omega_{0}\right\}, \\
& \alpha_{M}^{s}=\min _{a \geq 0}\left\{\alpha \in \mathbb{R}: \Omega_{0} \subseteq \alpha \tau \mathcal{B}^{n}\right\},
\end{aligned}
$$

and $\rho=\alpha_{m}^{s} / \alpha_{M}^{s}$. Note that $\rho \in(0,1)$. Defining also $K=$ $\min _{k \in \mathbb{N}}\left\{k \leq 1: c \lambda^{k} \leq \rho\right\}$, it follows that

$$
\left\|x_{K+i}^{\nu}\right\| \leq c \lambda^{K+i}\left\|x_{0}\right\| \leq \rho\left\|x_{0}\right\|, \quad \forall i \in \mathbb{N}_{N}
$$

with $r_{0}=s$ and $r_{K+i}^{\nu}=s$ for at least a $i \in \mathbb{N}_{N}$, from recurrence. This implies that there exists $i \in \mathbb{N}_{N}$ such that

$$
\forall(x, s) \in\left(\rho^{-1} \mathcal{B}^{n}\right) \times s \Rightarrow\left(x_{K+i}^{\nu}, r_{K+i}^{\nu}\right) \in \mathcal{B}^{n} \times s,
$$

that is equivalent, from homogeneity, to

$$
\forall(x, s) \in\left(\alpha_{M}^{s} \mathcal{B}^{n}\right) \times s \Rightarrow\left(x_{K+i}^{\nu}, r_{K+i}^{\nu}\right) \in\left(\alpha_{m}^{s} \mathcal{B}^{n}\right) \times s .
$$

Initializing Algorithm 1 with $\Omega_{0}$, which is such that $\alpha_{m}^{s} \mathcal{B}^{n} \subseteq$ $\Omega_{0}$, and iterating $K+N$ steps one obtains a set that contains all the points $(x, r)$ that can be stirred in $\alpha_{m}^{s} \mathcal{B}^{n} \times s$ by means of $\nu$ in $K+N$ steps or less. Therefore, from (14), we have

$$
\left(\alpha_{M}^{s} \mathcal{B}^{n}\right) \times s \subseteq \bigcup_{j \in \mathbb{N}_{N+K}}\left(\Omega_{j}^{s, s} \times s\right),
$$

which implies, from $\Omega_{0} \subseteq \tau \alpha_{M}^{s} \mathcal{B}^{n} \subseteq \operatorname{int}\left(\alpha_{M}^{s} \mathcal{B}^{n}\right)$, that the stop condition (5) is satisfied.

The meaning of Theorem 1 is that there is a stabilizing control policy $\nu(x, r)$ such that $\nu \in L(\mathcal{A})$ and the automaton state gets the value $s$ after $N$ steps at most, if and only if Algorithm 1 terminates in a finite number of iterations.

Remark 3: From (7) and the geometrical meaning of $\Omega^{s}$, it follows that every state $(x, s) \in \mathbb{R}^{n} \times s$ with $x \in \partial \Omega^{s}$ can be steered in $\rho \Omega^{s} \times s$ in $N^{s}$ or less steps. This means that there exists $n^{s}(x) \in \mathbb{N}_{N^{s}}$ such that

$$
\Psi_{\Omega^{s}}\left(x_{n^{s}(x)}^{\nu}\right) \leq \rho \Psi_{\Omega^{s}}(x)
$$

and $r_{n^{s}(x)}^{\nu}=s$, for an admissible $\nu \in L(\mathcal{A})$. Note that $(15)$ holds for every $x$, not only those on $\partial \Omega^{s}$, from homogeneity. Nevertheless, $\Psi_{\Omega^{s}}(x)$ does not necessarily decrease along the trajectory at times $k<n^{s}(x)$, nor for every $k>n^{s}(x)$, and then another gauge functional, the one of $\Theta^{r, s}$, has been built such that it is decreasing at every step.

Remark 4: If the Algorithm 1 terminates in finite time then every initial state $(x, r) \in \mathbb{R}^{n} \times \mathcal{R}$ can be exponentially stabilized. In fact, by Assumption 2 and Remark 1, the automaton state $s$ can considered as an initial state and the recurrent ECLF, being the gauge function of a $\mathrm{C}^{*}$-set, is defined on the whole $\mathbb{R}^{n}$, see Property 1 .

Remark 5: Given a recurrent ECLF, different control strategies can be designed for it to be decreasing along the 
trajectories. The one employed in the proof of Theorem 1 assures that the automaton state reaches $s$ every $N^{s}$ steps at most, by construction. Another classical approach consists in minimizing the ECLF value, i.e. selecting

$$
h^{r, s}(x)=\arg \min _{j \in \mathbb{N}} \Psi_{\Theta_{j}^{r, s}}(x)
$$

and applying the mode $i$ and the state in $m$ that generate $\Theta_{h^{r, s}}^{r, s}$ from the state $\Lambda_{h^{r, s}-1}^{s}$ in the Algorithm 1. Note that this control strategy does not assure the recurrence in $s$.

Thus, the existence of a stabilizing switching policy generating recurrent automaton trajectories is sufficient for stabilizability and can be obtained in finite time if it exists.

\section{EXAMPLES AND COUNTEREXAMPLES}

In the previous section we proved that the existence of a recurrent ECLF is sufficient for stabilizability. But what about necessity? Is it possible to have an ECLF and a related stabilizing switching control sequence that does not generate trajectories, that pass through one automaton state every $N$ steps at most? If we could prove that such sequences do not exist, necessity would result. But it is not the case.

Example 1: Consider a switching systems with two modes and the automaton given in Figure 1, left. Note that, in practice, every state is associated to the last mode activated and every possible sequence of modes is admissible. The matrices associated to the two modes are equal and Schur, that means that every switching sequence exponentially stabilizes the system, trivially. On the other hand, a switching sequence can be constructed such that no state is reached recurrently with a period that is bounded. Consider for instance the mode sequence $\{1,2,1,1,2,2,1,1,1,2,2,2,1, \ldots\}$ generating the automaton trajectory $\{a, a, b, a, a, b, b, a, a, a, b, b, b, a, \ldots\}$, with initial state $a$. The sequence of delays for the trajectory to reach the state $a$ (and analogously for $b$ ) is $\{1,2,1,3,1,1,4, \ldots\}$ whose upper bound is not bounded.

Therefore, there might exist stabilizing switching sequences generated by an automaton that do not provide recurrent trajectories. On the other hand, this does not mean that there is not another switching sequence generating recurrent trajectories, as for the case in Example 1.
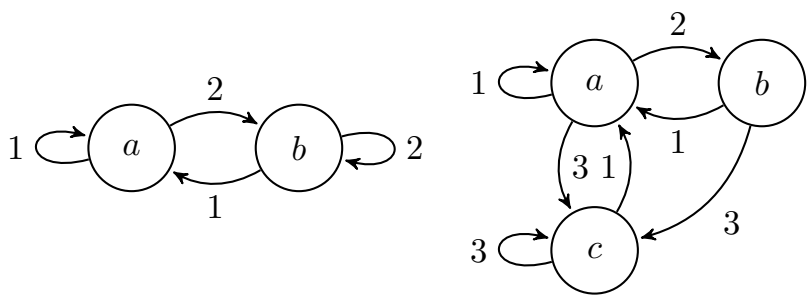

Fig. 1. Automata of Example 1, left, and Example 2, right.

Moreover, one may wonder if there is a relation between the recurrent ECLFs and the periodic switching control sequences, i.e. composed by a finite sequence of modes, not dependent on the state, that repeats cyclically in time.

Example 2: To construct a counterexample, and to have some insight on the problem, we considered the Example
17 in the paper [6]. Consider a switched system with three modes and no constraints on the mode sequences. The matrices are

$$
A_{1}=A R(0), \quad A_{2}=A R\left(\frac{2 \pi}{3}\right), \quad A_{3}=A R\left(\frac{-2 \pi}{3}\right),
$$

where

$$
A=\left[\begin{array}{cc}
a & 0 \\
0 & a^{-1}
\end{array}\right], \quad R(\theta)=\left[\begin{array}{cc}
\cos (\theta) & -\sin (\theta) \\
\sin (\theta) & \cos (\theta)
\end{array}\right],
$$

with $a=0.6$. Consider the set $\Omega_{0}=\mathcal{B}^{2}$. As proved in [6], the stabilizability geometric condition [6] holds at the first step. Moreover, we know from [6] that no periodic stabilizing switching law exists. This would not mean that periodic sequences cannot be generated, but the fact that there is not one periodic stabilizing switching law that does not depend on the state. Roughly speaking, there is not an open-loop (then periodic) stabilizing switching law for this system. We consider now the deterministic automaton given by three states, $a, b$ and $c$, such that every mode can be selected at every instant and the successor is $a$ when the mode 1 is applied, is $b$ for 2 and $c$ for 3 . Thus, once more, every possible sequence of modes can be generated and every automaton mode represents the last mode applied. Since no periodic switching law stabilizes the system, then,
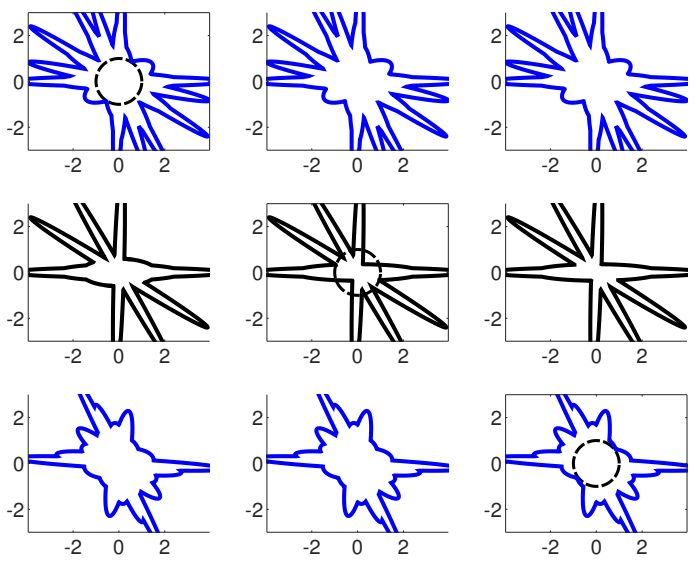

Fig. 2. Sets $\Omega^{r, s}$ for all $r, s \in \mathcal{I}$ and $N^{s}=4$. The set $\Omega^{r, s}$ is depicted in the line $s$ column $r$. In blue the sets related to recurrent ECLF.

in principle, there is no need for a stabilizing sequence to reach at least one state every $N$ steps at most, with $N \in \mathbb{N}$. Note in fact that if a periodic sequence stabilizes the system then, by its nature, it must pass through every state related to the modes in the sequence with a delay smaller than or equal to the cycle length. Then, in this case, the Algorithm 1 would terminate in finite time.

So, the question we would like to answer to is: provided a periodic switching law does not exist, does there exist a stabilizing sequence that pass through one state $s$ with bounded delay, hence recurrent, for every $x$ ? The answer is yes. In fact by applying Algorithm 1 to every mode in $\mathcal{I}$ we found that the stop condition holds. Moreover, we proved that also by removing two arcs, i.e. forbidding two transitions, see Figure 1 right, two different recurrent ECLF exist with 
$N^{s}=4$, one recurrent in the mode $a$, one in the mode $c$, see Figure 2.

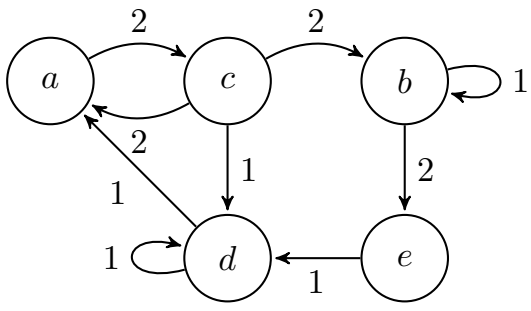

Fig. 3. Automaton of Example 3.

Example 3: Finally, we apply our algorithm to a 2dimensional switched system with 2 unstable modes

$$
A_{1}=\left[\begin{array}{cc}
1.2 & 0 \\
0 & 0.5
\end{array}\right], \quad A_{2}=1.1 R(\pi / 3),
$$

and constraints determined by the 5-states automaton of Figure 3. The Algorithm 1 has been applied for every $s \in \mathcal{I}$. No recurrent ECLF has been obtained after 5 steps, but for $N^{s}=6$ the stop condition holds for three automaton states, i.e. for $a, c$ and $d$. The resulting sets are drawn in Figure 4. The control policy related to the mode $s=$ $d$ has been applied, resulting in the state and automaton trajectories shown in Figure 5. Note that, since we applied a min-switching control strategy, sketched in Remark 5, the recurrence in $d$ is not assured every 6 steps or less, see the instants between 17 and 24 .
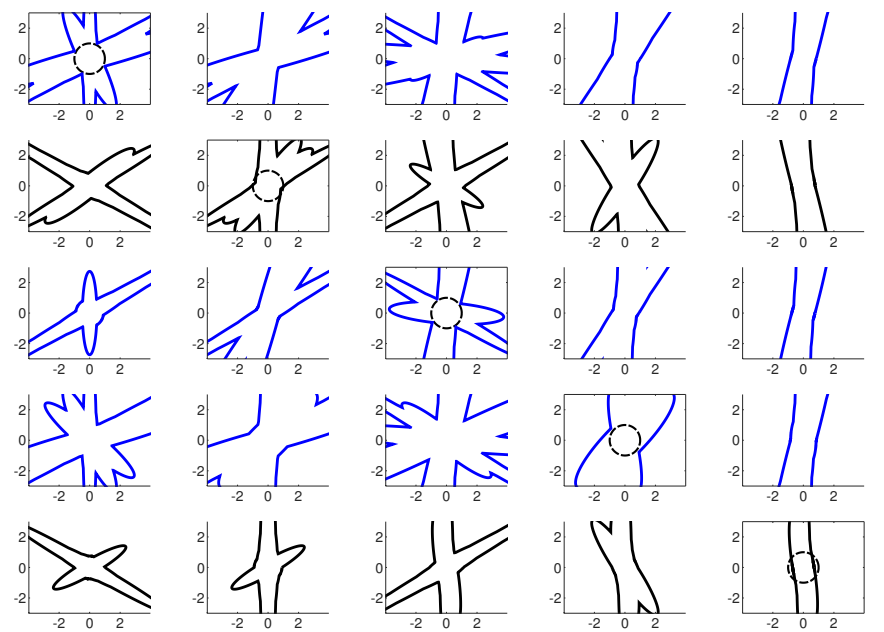

Fig. 4. Sets $\Omega^{r, s}$ for all $r, s \in \mathcal{I}$ and $N^{s}=6$. The set $\Omega^{r, s}$ is depicted in the line $s$ column $r$. In blue the sets related to recurrent ECLF.

\section{CONCLUSions}

In this paper we presented a constructive approach to characterize a class of exponential control Lyapunov functions for switched linear systems subject to constraints on the switching law. The method is based on an algorithm whose finite termination provides a necessary and sufficient condition for the existence of a recurrent ECLF. A preliminary analysis of the relations between this class of ECLF and general stabilizability is given.
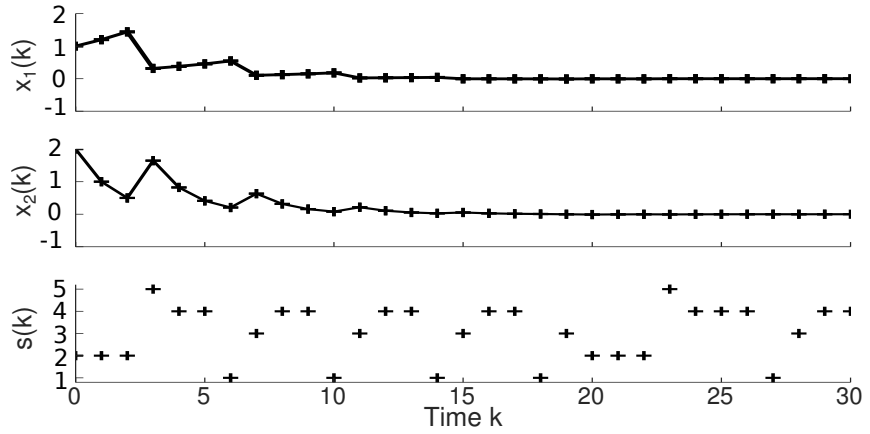

Fig. 5. Evolutions of the systems states, top and middle, and automaton state, bottom, where 1 denotes $a ; 2 b ; 3 c ; 4 d$ and $5 e$.

\section{REFERENCES}

[1] A. A. Ahmadi, R. M. Jungers, P. A. Parrilo, and M. Roozbehani. Joint spectral radius and path-complete graph Lyapunov functions. SIAM Journal on Control and Optimization, 52(1):687-717, 2014.

[2] C. Baier, J.-P. Katoen, et al. Principles of model checking, volume 26202649. MIT press Cambridge, 2008.

[3] F. Blanchini. Nonquadratic Lyapunov functions for robust control. Automatica, 31:451-461, 1995.

[4] F. Blanchini and S. Miani. Set-Theoretic Methods in Control. Birkhäuser, 2008.

[5] F. Blanchini and C. Savorgnan. Stabilizability of switched linear systems does not imply the existence of convex Lyapunov functions. Automatica, 44:1166 - 1170, 2008.

[6] M. Fiacchini, A. Girard, and M. Jungers. On the stabilizability of discrete-time switched linear systems: Novel conditions and comparisons. IEEE Transactions on Automatic Control, PP(99):1-1, 2015.

[7] M. Fiacchini and M. Jungers. Necessary and sufficient condition for stabilizability of discrete-time linear switched systems: A set-theory approach. Automatica, 50(1):75 - 83, 2014.

[8] J. C. Geromel and P. Colaneri. Stability and stabilization of discretetime switched systems. International Journal of Control, 79(7):719728 , July 2006.

[9] J.-W. Lee and G. E. Dullerud. Uniformly stabilizing sets of switching sequences for switched linear systems. IEEE Transactions on Automatic Control, 52(5):868-874, 2007.

[10] D. Liberzon. Switching in Systems and Control. Birkhäuser, Boston, MA, 2003.

[11] H. Lin and P. J. Antsaklis. Stability and stabilizability of switched linear systems: a survey of recent results. IEEE Transaction on Automatic Control, 54(2):308-322, 2009.

[12] P. Matthew and R. Jungers. Converse Lyapunov theorems for discretetime linear switching systems with regular switching sequences. arXiv preprint arXiv:1410.7197, 2014.

[13] A. P. Molchanov and Y. S. Pyatnitskiy. Criteria of asymptotic stability of differential and difference inclusions encounterd in control theory. Systems \& Control Letters, 13:59-64, 1989.

[14] A. M. Rubinov and A. A. Yagubov. The space of star-shaped sets and its applications in nonsmooth optimization. In V.F. Demyanov and L.C.W. Dixon, editors, Quasidifferential Calculus, pages 176-202. Springer Berlin Heidelberg, 1986.

[15] Z. Sun and S. S. Ge. Stability Theory of Switched Dynamical Systems. Springer, 2011.

[16] Y. Wang, N. Roohi, G. E. Dullerud, and M. Viswanathan. Stability of linear autonomous systems under regular switching sequences. In IEEE 53rd Annual Conference on Decision and Control (CDC), pages 5445-5450. IEEE, 2014.

[17] G. Weiss and R. Alur. Automata based interfaces for control and scheduling. In Hybrid Systems: Computation and Control, pages 601613. Springer, 2007.

[18] W. Zhang, A. Abate, J. Hu, and M. P. Vitus. Exponential stabilization of discrete-time switched linear systems. Automatica, 45(11):25262536, 2009. 